\author{
David R. Foran, ${ }^{1}$ Ph.D.; Michael E. Gehring, ${ }^{2, \dagger}$ M.S.; and Shawn E. Stallworth ${ }^{3}$
}

\title{
The Recovery and Analysis of Mitochondrial DNA from Exploded Pipe Bombs*
}

\begin{abstract}
Improvised explosive devices (IEDs) represent one of the most common modes of arbitrarily injuring or killing human beings. Because of the heat generated by, and destruction to, an IED postconflagration, most methods for identifying who assembled the device are ineffective. In the research presented, steel pipe bombs were mock-assembled by volunteers, and the bombs detonated under controlled conditions. The resultant shrapnel was collected and swabbed for residual cellular material. Mitochondrial DNA profiles were generated and compared blind to the pool of individuals who assembled the bombs. Assemblers were correctly identified $50 \%$ of the time, while another $19 \%$ could be placed into a group of three individuals with shared haplotypes. Only one bomb was assigned incorrectly. In some instances a contaminating profile (mixture) was also observed. Taken together, the results speak to the extreme sensitivity the methods have for identifying those who assemble IEDs, along with precautions needed when collecting and processing such evidence.
\end{abstract}

KEYWORDS: forensic science, forensic mitochondrial DNA, improvised explosive device, nested PCR

In recent years, terrorism - the indiscriminant and generally nonmilitary taking of human life-has become a priority in the American awareness. In over $70 \%$ of terrorist incidents explosives are used, most commonly in the form of improvised explosive devices (IEDs; 1). Of these, approximately half are pipe or tube bombs (2), which have been involved in a range of widely documented terrorist incidents, including the Olympic Park bombings in Atlanta and the Unabomber attacks, among others (1,3). Pipe bombs, like most IEDs, are easy to construct out of readily available materials, and can be quite effective in both maiming and intimidating individuals.

One of the major problems facing law enforcement agencies in addressing these incidents is that most explosive devices are left on a time delay and leave behind limited forensic evidence. It is therefore very difficult to identify possible suspects or link individuals to the device based on the physical evidence left at the scene. Currently, physical characteristics of the device (bomb fragments remaining following detonation, the type of explosive used, etc.) may allow investigators to trace components back to specific suppliers, providing class evidence that might relate to a suspect. Beyond this, fragments of the device can be examined for fingerprints. However, such individualizing evidence is rarely obtained given the damage deflagration causes.

With the advances made in forensic biology, analysis of DNA now has the potential to lead to the identification of the manufacturer of an IED. Brief contact between a person and object can leave behind sufficient DNA for analysis and potential

${ }^{1}$ Forensic Science Program, School of Criminal Justice and Department of Zoology, Michigan State University, East Lansing, MI.

${ }^{2}$ Forensic Science Program, School of Criminal Justice, Michigan State University, East Lansing, MI.

${ }^{3}$ Bomb Squad, Forensic Science Division, Michigan State Police, Lansing, MI.

†Present address: Georgia Bureau of Investigation, Forensic Biology, Decatur, GA.

*A portion of this work was presented at the American Academy of Forensic Sciences Annual Meeting in San Antonio, TX, February 22, 2007.

Received 9 Sept. 2007; and in revised form 26 Apr. 2008; accepted 26 Apr. 2008. identification (4, reviewed in 5). Further, there have been at least two studies of short tandem repeat (STR) analysis from detonated IEDs $(6,7)$. In both cases some STR data were generated, though only limited sample sizes were tested ( $n=20$ and $n=5$, respectively), and of these, a full nuclear DNA profile was obtained once, as were a few partial profiles.

The primary factor influencing successful DNA-based analysis of IEDs is the quality of DNA recovered. The conflagration of an explosive device generates large amounts of heat, which results from the burning of fuel as well as from metal fatigue if a metallic vehicle is used. The heat can be detrimental to the integrity of any DNA present, and it is probable that recovered DNA will be highly fragmented. In addition, only a small number of cells, most likely dead epithelia, will generally be transferred during contact between a person and the IED, meaning predetonation DNA levels are already low.

Poor genetic outcomes resulting from small amounts of degraded DNA can be circumvented in various ways. First, mitochondrial DNA (mtDNA) analysis may be attempted. Because of its higher copy number, from hundreds to thousands of copies per cell (8), its relative robustness (9), and the ability to obtain it from compromised samples (reviewed in 10), mtDNA analysis has the potential to be more successful when analyzing evidence exposed to harsh conditions. For example, mtDNA has long been known to be extractable from hair, fingernails, and ancient bone when nuclear DNA cannot be recovered (e.g., 11). In addition, as mtDNA is maternally inherited, a reference sample can be obtained not only from the suspect, but also from any maternally related family member. The disadvantage of mtDNA analysis is a loss of individualizing power, though this might be offset by more consistently obtaining results from compromised samples when compared with STR analyzes. The problem of DNA degradation that is likely to occur under the conditions of a bomb blast also has the potential of being overcome using mtDNA; because it is analyzed through sequencing, small overlapping fragments can be used to construct a complete haplotype.

The limited amount of DNA that is expected to be recovered from a detonated IED may be addressed by increasing PCR cycle number, although this often leads to the production of extraneous 
PCR products, making subsequent analysis impossible. Amplification of nontarget DNA can be prevented using nested PCR (12), in which two rounds of PCR are conducted with two different sets of primers, the second set internal to the first. Because the internal (nested) primers are unlikely to anneal anywhere other than the target sequence resulting from the first round of amplification, an increased number of PCR cycles can be employed while minimizing the chances of nontarget product being synthesized. The drawback of nested PCR is that extra manipulation of a sample is required, which can potentially lead to problems with contamination.

In the blind study presented here, the feasibility of identifying persons who assembled pipe bombs was examined through mtDNA analysis. Subjects handled sterilized pipe bomb components that were subsequently assembled and detonated. Exploded fragments were collected and swabbed, mtDNA was amplified via seminested PCR, and the resulting DNA sequences were determined. Comparisons among mtDNA sequences obtained from bombs and a set of reference sequences were used to assign bombs to subjects.

\section{Materials and Methods}

\section{Sample Decontamination and Preparation}

Pipe bombs were assembled using a 1-foot galvanized steel pipe nipple of 1-inch diameter and two 1-inch diameter galvanized steel end caps (obtained from local hardware stores), a length of safety fuse (2 or 4 feet), and a Thermolite Connector ignition cap (Ensign Bickford, Simsbury, CT). Pipes and end caps were soaked in a $10 \%$ bleach solution for $1 \mathrm{~h}$, rinsed with distilled water, and placed in a Spectrolinker XL-1500 UV Crosslinker (Spectronics Corporation, Westbury, NY) for $10 \mathrm{~min}$, turning halfway through for complete exposure, to remove exogenous DNA (6). The fuse and Thermolite Connector ignition cap were wiped with a $10 \%$ bleach solution. Fuses were affixed via a hole drilled in one end cap and secured with hot glue. Bombs were sealed in paper bags and randomly selected by subjects. Eighteen individuals participated in the study, and a total of 51 bombs were prepared and detonated. Thirteen bombs were used in protocol development; the results from those processed using the resultant standardized protocol are presented here. Subjects handled two bombs each, with two exceptions-one handled a single bomb while another handled three. Two control bombs were included, which were unhandled but otherwise processed like the others. All testing was carried out as per University Institutional Review Board stipulations.

In simulating pipe bomb construction, subjects handled the bombs by screwing and unscrewing the end caps for a total of $30 \mathrm{sec}$, as well as providing buccal swabs for reference DNA. All subject and bomb samples were anonymized and the remainder of the experiment was performed blind. Latex gloves were worn by the three investigators who handled/processed the bombs. Immediately before detonation, pipes were filled 3/4 with SR 4756 (IMR Powders, Shawnee Mission, KS), a single-base, smokeless gunpowder. Bombs were detonated in the Lansing, MI Fire Fighter Training Facility smoke room. All visible bomb fragments were collected by the remaining two investigators using a sweep of the room, and were stored in paper bags at room temperature until processing. Owing to the amount of smoke and soot in the air, particulate filter masks were worn during the second half of collections.

\section{DNA Isolation and Purification}

Buccal swabs and pipe bomb sample DNAs were processed separately to prevent cross-contamination. External bomb fragment surfaces still retaining zinc coating were double swabbed with a sterile cotton swab moistened with $100 \mu \mathrm{L}$ of digestion buffer (20 mM Tris, $50 \mathrm{mM}$ EDTA, 0.1\% SDS, pH 7.5), followed by a dry cotton swabbing (13). Only nonswabbed sides of bomb fragments were handled. Both swab ends were placed into a microcentrifuge tube with $400 \mu \mathrm{L}$ of digestion buffer and $6 \mu \mathrm{L}$ of $20 \mathrm{mg} / \mathrm{ml}$ proteinase $\mathrm{K}$. Tubes were vortexed, briefly centrifuged, and incubated overnight at $56^{\circ} \mathrm{C}$. Swab tips were then placed in spin baskets, centrifuged at 14,000 rpm $(21,000 \times g)$ for $5 \mathrm{~min}$ and discarded. Buffer collected from the swabs was extracted using an equal volume of phenol:chloroform:isoamyl alcohol (25:24:1), followed by an equal volume of chloroform. The aqueous layer was transferred to a Microcon YM-100 spin column (Millipore, Billerica, MA) and centrifuged at $500 \times g$ for $20 \mathrm{~min}$, followed by two washes of $300 \mu \mathrm{L}$ TE (10 mM Tris, $1 \mathrm{mM}$ EDTA, pH 7.5). Samples were resuspended in $20 \mu \mathrm{L}$ of $\mathrm{TE}$ and stored at $-20^{\circ} \mathrm{C}$.

\section{Sample Amplification and Sequencing}

Three mtDNA regions were amplified from each pipe bomb sample using semi-nested PCR and published primers (14). The first section of hyper-variable region 1 (HV1), denoted HV1-1, was amplified with primers F15989 and R16322, and subsequently with F15989 and R16281 for semi-nested PCR. HV1-2 was amplified with F16144 and R16410, and subsequently with F16190 and R16410 for semi-nested PCR. Hyper-variable region 2 (HV2) was amplified using F82 (5'-ATAGCATTGCGAGACGCTGG-3') and R484, and subsequently with F155 and R484 for semi-nested PCR. The thermocycling program was: $2 \mathrm{~min}$ at $94^{\circ} \mathrm{C}$, followed by 38 cycles of $30 \mathrm{sec}$ at $94^{\circ} \mathrm{C}, 1 \mathrm{~min}$ at $60^{\circ} \mathrm{C}$, and $1 \mathrm{~min}$ at $72^{\circ} \mathrm{C}$. Amplifications using primer $\mathrm{R} 484$ followed the above parameters, except substituting a $45^{\circ} \mathrm{C}$ annealing temperature. Reactions were carried out in $20 \mu \mathrm{L}$ volumes, using $0.5 \mu \mathrm{L}$ of template, primers at $2 \mu \mathrm{M}, 0.5 \mathrm{mg} / \mathrm{ml}$ deacetylated BSA, $2.5 \mathrm{mM} \mathrm{MgCl}_{2}, 200 \mu \mathrm{M}$ dNTPs, and Taq polymerase (Promega, Madison WI) at 1 unit/reaction. Reference samples were amplified with primers F15989 and R569, using the same parameters and 32 amplification cycles. PCR negative and positive controls were run with both standard and nested PCR. Five microliters of PCR product was electrophoresed on a $1.5 \%$ agarose gel and stained with ethidium bromide. A seminested PCR reaction was carried out for any bomb sample that produced a light band or no band at all. This was performed using $1 \mu \mathrm{L}$ of the initial PCR reaction for template and the same amplification parameters as the first round, for 24 cycles.

PCR products were purified on Microcon-100 spin columns as described above and sequenced with a CEQ DTCS Quick Start Kit and a CEQ8000 genetic analyzer (Beckman Coulter, Fullerton, CA) using the manufacturer's protocol, in a reaction volume of $10 \mu \mathrm{L}$. The primers for semi-nested PCR were also employed in sequencing reactions, while reference samples were sequenced with F15989, R16410, F15, and R569. A capillary separation time of 45 min was used for HV1 samples and 60 min for HV2 and reference samples. Sequences were aligned and proofread using BioEdit Sequence Alignment Editor (15). DNAs were characterized based on differences from the human reference sequence (16); these were compared to make donor assignments.

\section{Results}

\section{Bomb Fragmentation}

Fragmentation of the pipe bombs ranged from little fragmentation of the end caps to complete destruction of the pipe (Fig. 1). 


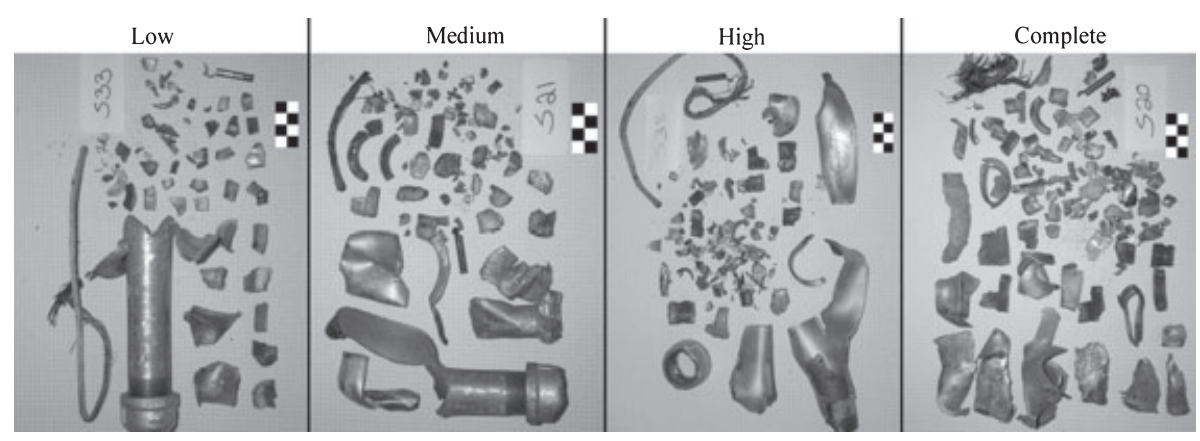

FIG. 1-Bomb fragmentation. The fragmentation of the bombs varied widely, from very few fragments and the body of the bomb largely intact, to completely fragmented. Size scale is indicated by the checkered marker, with each square measuring $1 \mathrm{~cm}$.

Fragment sizes as small as $2 \mathrm{~mm}$ were collected. Higher levels of fragmentation resulted in less useable surface area for DNA recovery because of abraded edges that could not be swabbed. Although the room was closed during detonation, in some instances the door or ceiling vent were blown open and fragments were propelled out of the confines of the room. These were discarded to prevent possible cross-contamination.

\section{MtDNA Amplification, Sequencing, and Donor Assignation}

Initial PCR amplification yielded sufficient product for sequencing from 15 of the 38 samples for HV1-1, five of 38 samples for HV1-2, and no samples for HV2. Semi-nested PCR of all samples with insufficient first-round amplicon production generated enough DNA for sequencing.

Sequences were obtained from all bombs (Table 1), with an average read length of 256 bases for HV1 $(n=38)$ and 283 bases for HV2 $(n=22)$. Eighteen of the 36 subject samples $(50 \%)$ were correctly assigned to a single donor, seven (19\%) were correctly narrowed to groups of three donors who had shared haplotypes,

TABLE 1-Summary of sample assignations.

\begin{tabular}{lcc}
\hline Donor Assignment & Number of Samples & Percentage \\
\hline Assignable to single donor & 18 & 50 \\
Assignable to a subset of donors & $7 *$ & 19.4 \\
Not assignable & 10 & 27.8 \\
Incorrectly assigned & 1 & 2.8 \\
\hline
\end{tabular}

*In cases where an assignment was made to a subset of donors, three donors matched the sample. while $10(28 \%)$ could not be assigned. A single sample was assigned to the wrong subject. Correct sequence data were recovered for 16 of the 18 subjects from at least one bomb and seven had correct sequence results from both of their bomb samples.

HV1 amplification of the two control bomb samples produced amplicons; however their sequencing resulted in "nonsense" data that could not be aligned with the human reference sequence. Sixteen of 36 test samples had indications of a mixed profile, with a small number of sites containing two clear bases. In 13 instances, mixtures were consistent with a test subject and one of the investigators who handled the bombs before or after detonation: two from the investigator who only handled the bombs preconflagration and two from the investigator who only collected bomb fragments, with the remainder at undetermined time points. While making subject assignments from mixtures is not a standard procedure in mtDNA casework, in the current blind study it was considered informative with regards to how contamination was manifested. From the 16 samples, six were correctly assigned based on clear major/minor donors at a small number of bases (Fig. 2), the former being a test subject and the latter an investigator. There was no relationship between mixed profiles and the use of nested PCR, with the percentage of mixtures being slightly lower in nested amplification products.

\section{Discussion \\ MtDNA Amplification and Subject Assignment}

The research presented here demonstrates that informative mtDNA profiles can be recovered from pipe bomb fragments, even after being subjected to the extreme conditions of low-yield

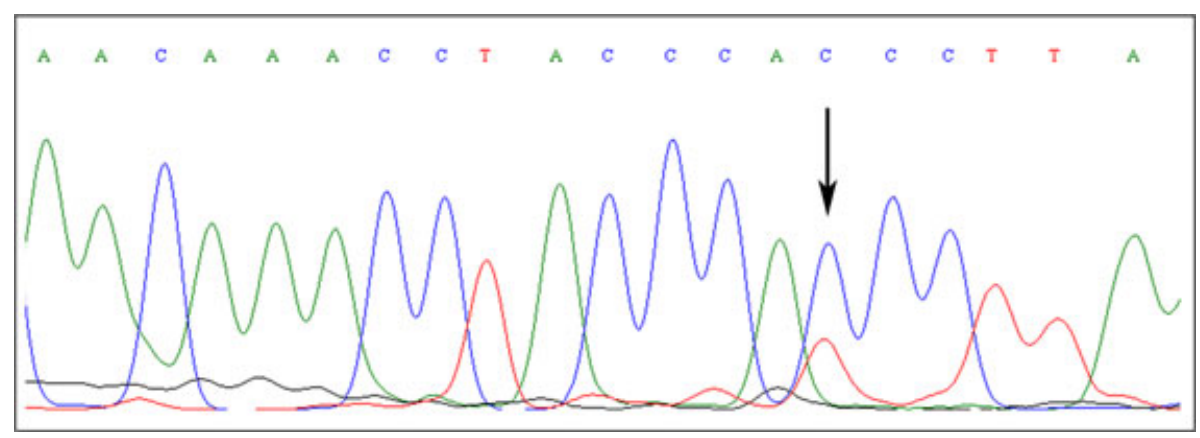

FIG. 2-Mixture analysis. In some instances mtDNA sequences showed the presence of more than one contributor. It was often possible to distinguish and subtract a minor contributor (arrow) and thus deduce the haplotype of the bomb handler, given the closed population of test subjects. The minor contribution was consistent with one of the investigators in most instances. 
explosive conflagration. Two-thirds of the bombs in this blind study were correctly assigned, and the individualizing success rate, given the closed population, was $50 \%$, a notable improvement on the 5\% success rate in analysis of STRs (6). The increase likely stems from multiple factors, including the higher cellular copy number of mtDNA when compared with the single copy loci used in most forensic analyses (8), the differential degradation of mitochondrial and nuclear DNA (9), and the smaller amplicon size that can be utilized for mtDNA analysis. In this study, the majority of bomb samples were successfully typed following nested PCR. Likewise, the benefit of assaying overlapping mtDNA fragments (17) was apparent in the higher sequencing success rate for HV1 (256 bp, $n=36$ ), which was amplified as two separate fragments, compared with HV2 (283 bp, $n=22)$, amplified as a single fragment.

\section{Advantages and Caveats}

A major consideration with mtDNA analysis of touch samples is its extreme sensitivity. The short amplicons and nested PCR methodology used here allowed DNA to be amplified and analyzed from every swabbed bomb, and two-thirds of the IEDs were correctly assigned, a substantial increase in success over standard STR testing (6). Furthermore, only one assay in 36 led to a mis-assigned bomb, wherein the haplotype obtained was shared with one of the subjects. It is not clear when or how this occurred, and owing to the anonymity of the testing it was not possible to investigate if that person might have been in the laboratory during any stage of bomb preparation or processing.

Just as important to note is that mtDNA mixtures were sometimes observed, most of which could have originated from a subject and one of the three investigators who handled/processed the bombs before and/or after detonation. The most frequent of these was consistent with the investigator who handled the bombs the most, including predetonation decontamination, collection and swabbing of fragments, and subsequent DNA analyses. Also observed were probable mixtures resulting from an investigator who had only preconflagration contact with bombs, while filling them with smokeless powder, placing them, and lighting fuses. Although gloves were worn during this procedure, a mask was not, which may have resulted in the observed outcome. Finally, mtDNA results consistent with the third investigator, who solely helped to collect bomb fragments, were observed. Again, this person wore gloves, but no mask, during bomb fragment collection. In the three remaining instances of apparent contamination no source could be determined. These may have originated from members of the bomb squad and fire department on the scene during detonations, persons in the laboratory, or the swabs themselves; we continue to investigate all possibilities. Taken together, the results emphasize the extreme care that will need to be employed when genetically analyzing postdetonation bomb evidence, particularly, it seems, during the difficult evidence collection stage.

\section{Conclusions}

The results of this study demonstrate the value of mtDNA analysis in identifying the manufacturers of IEDs under postconflagration conditions. Not only were half of the detonated pipe bombs assignable to a single individual, and two-thirds correctly assigned a haplotype, in almost all instances recoverable mtDNA remained after conflagration, an obvious improvement over nuclear DNA markers. Even with low DNA quantities and high levels of degradation, analysis of mtDNA was possible utilizing nested PCR and sequence construction from multiple fragments. Important to note however, is that while this approach is extremely sensitive, owing to its sensitivity it is particularly susceptible to contamination from outside DNA sources. As such, extensive precautions will need to be taken not only in the laboratory (where, advantageously, controls can be included to help recognize if contamination occurs), but also at the scene, where the luxury of running controls may be impractical or impossible. Other factors, such as the level of bomb fragmentation and the bomb making materials, will likely influence both the success of analyses and range of difficulties encountered. However, despite shortcomings that need to be considered, the results presented here show that exclusion of large numbers of suspects, or identification from a select pool of suspects, may be achieved using this approach. Our preliminary study into mtDNA identification of those who assemble IEDs holds more promise than any technique that has preceded it, and as such helps form a foundation for further research and legal precedence using genetic methods.

\section{Acknowledgments}

The authors gratefully acknowledge the help and support of Amy Barber and Stefanie Kremer of the Michigan State University Forensic Science Program, members of the Michigan State Police Bomb Squad, and Captain Trent Atkins and members of the Lansing Fire Department. M. Gehring was supported through a Department of Homeland Security graduate fellowship.

\section{References}

1. Burke R. Counter-terrorism for emergency responders. Boca Raton, Florida: CRC Press LLC, 2000.

2. 2004 third quarter statistical report. The Arson and Explosives National Repository Branch Report. Available at: http://www.interfire.org/ res_file/natlrep.asp (accessed January 3, 2008 [restricted access]).

3. Schweitzer GE, Dorsch CC. Modern mutations of global terrorism. The Bridge 1998;28:1-9.

4. Van Oorschot RAH, Jones MK. DNA fingerprints from fingerprints. Nature 1997;387:767.

5. Wickenheiser RA. Trace DNA: a review, discussion of theory and application of the transfer of trace quantities of DNA through skin contact. J Forensic Sci 2002;47:442-50.

6. Esslinger KJ, Siegel JA, Spillane H, Stallworth S. Using STR analysis to detect human DNA from exploded pipe bomb devices. J Forensic Sci 2004;49:481-4.

7. Bechaz H, The Australian Federal Police. The profiling of DNA of fired cartridge cases and pipe bomb fragments [Bachelor's thesis]. Canberra, Australia: University of Canberra, Canberra, Australian Capital Territory, Australia, 2001.

8. Robin E, Wong R. Mitochondrial DNA molecules and virtual number of mitochondria per cell in mammalian cells. J Cell Physiol 1988;136:50713.

9. Foran DR. The relative degradation of nuclear and mitochondrial DNA: an experimental approach. J Forensic Sci 2006;51:766-70.

10. Holland MM, Parsons TJ. Mitochondrial DNA sequence analysis-validation and use for forensic casework. Forensic Sci Rev 1999;11:21-51.

11. Merriwether DA, Rothhammer F, Ferrell RE. Genetic variation in the new world: ancient teeth, bone, and tissue as sources of DNA. Experientia 1994;50:592-601.

12. Strom CM, Rechitsky S. Use of nested PCR to identify charred human remains and minute amounts of blood. J Forensic Sci 1998;43:696700 .

13. Sweet D, Lorente M, Lorente JA, Valenzuela A, Villanueva E. An improved method to recover saliva from human skin: the double swab technique. J Forensic Sci 1997;42:320-2.

14. Edson SM, Ross JR, Coble MD, Parsons TJ, Barritt SM. Naming the dead-confronting the realities of rapid identification of degraded skeletal remains. Forensic Sci Rev 2004;16:64-89. 
15. Hall TA. BioEdit: a user-friendly biological sequence alignment editor and analysis program for Windows 95/98/NT. Nucleic Acids Symp Ser 1999;41:95-8.

16. Anderson S, Bankier AT, Barrell BG, de Bruijn MH, Coulson AR, Drouin J, et al. Sequence and organization of the human mitochondrial genome. Nature 1981;290:457-65.

17. Gabriel MN, Huffine EF, Ryan JH, Holland MM, Parsons TJ. Improved mtDNA sequence analysis of forensic remains using a "mini-primer set" amplification strategy. J Forensic Sci 2001;46:247-53.
Additional information and reprint requests:

David R. Foran, Ph.D.

School of Criminal Justice and Department of Zoology

Michigan State University

560 Baker Hall

East Lansing

MI 48824

E-mail: foran@msu.edu 